\title{
EFECTO DE LA LIMPIEZA SANITARIA DE CORMOS DE PLÁtANO (Musa AAB SIMMONDS) SOBRE NEMATODOS FITOPARÁSITOS
}

\section{EFFECT OF SANITARY CLEANNESS OF PLANTAIN (Musa AAB SIMMONDS) CORMS ON PLANT PARASITIC NEMATODES}

\author{
Óscar Adrián Guzmán1, Jairo Castaño², Bernardo Villegas³
}

\begin{abstract}
1,2,3 Programa de Maestría en Fitopatología. Facultad de Ciencias Agropecuarias. Universidad de Caldas, Manizales, Caldas. ${ }^{1}$ Profesor Auxiliar, Universidad de Caldas. Correo electrónico: oscar.guzman@ucaldas.edu.co 2 Profesor Titular, Universidad de Caldas. Correo electrónico: jairo.castano_z@ucaldas.edu.co ${ }^{3}$ Profesor Asistente, Universidad de Caldas. Correo electrónico: bernardo.villegas@ucaldas.edu.co
\end{abstract}

Rev. U.D.C.A Act. \& Div. Cient. 15(1): 87 - 95, 2012

\section{RESUMEN}

El empleo de semilla vegetativa de banano y de plátano favorece la diseminación de nematodos fitoparásitos, como Radopholus similis, Pratylenchus coffeae, Helicotylenchus multicinctus, $H$. dihystera, Meloidogyne incognita, M. javanica y Rotylenchulus reniformis. El objetivo de este estudio fue determinar el efecto de la limpieza sanitaria "pelado" de cormos de plátano Dominico-Hartón sobre el crecimiento de plántulas y de poblaciones de nematodos fitoparásitos en almácigo. Se utilizaron seis tratamientos, cada uno con 20 cormos, 40 con limpieza sanitaria "pelado" en suelo estéril con y sin inmersión en aldicarb; 40 cormos tradicionales (sin pelar), con y sin inmersión en aldicarb y 40 cormos con limpieza sanitaria "pelado", con y sin inmersión en aldicarb, en suelo infestado por fitonematodos. Los tratamientos fueron asignados en un diseño experimental completamente aleatorio. El mejor tratamiento fue el empleo de suelo esterilizado (libre de nematodos) y cormos con limpieza sanitaria "pelado", sin encontrarse diferencia estadística con el uso o no de aldicarb, ya que no se hallaron nematodos fitoparásitos, tanto en raíces como en suelo, después de 50 días; mientras que en los demás tratamientos, la cantidad de nematodos fitoparásitos osciló entre 143 y 2.495 individuos, en $100 \mathrm{~g}$ de raíces y entre 11 y 18 nematodos, en $100 \mathrm{~g}$ de suelo. Los resultados demostraron que la limpieza sanitaria "pelado" de cormos permite obtener plántulas libres de nematodos fitoparásitos, lo que favorece su buen crecimiento y desarrollo, práctica que permite llevar material sano al campo.
Palabras clave: Semilla, sanidad, musáceas, Radopholus similis.

\section{SUMMARY}

The use of vegetative seed of banana and plantain favor the dissemination of plant parasitic nematodes such as: Radopholus similis, Pratylenchus coffeae, Helicotylenchus multicinctus, $H$. dihystera, Meloidogyne incognita, M. javanica and Rotylenchulus reniformis. The objective of this study was to determine the effect of the sanitary cleanness "peeled" of corms of Dominico-Harton plantain on seedlings' development and plant parasitic nematodes population, in the nurseries. The assay included six treatments, each one, with 20 corms, distributed as follow: 40 corms with sanitary cleanness "peeled" in sterilized soil, with and without immersion in aldicarb; 40 traditional corms with and without immersion in aldicarb;and, 40 corms with sanitary cleanness "peeled" with and without immersion in aldicarb, in infested soil with plant parasitic nematodes. The treatments were assigned in a completely randomized experimental design. The best treatment was the use of sterilized soil (free of nematodes) and corms with sanitary cleanness, without having statistically differences using or not aldicarb, since was not found plant parasitic nematodes after 50 days, in comparison to the other treatments in which the population of nematodes ranged between 143 y 2,495 individuals on $100 \mathrm{~g}$ of roots and between 11 and 18 nematodes on $100 \mathrm{~g}$ of soil. The results demonstrated that the practice of the sanitary 
cleanness "peeled" of corms, allows producing seedlings free of nematodes, which favors good growth and development of the seedlings, permitting to bring clean planting material to the field.

Key words: Seed, health, musaceae, Radopholus similis.

\section{INTRODUCCIÓN}

El plátano, se siembra tradicionalmente por medio de material vegetativo o asexual, como cepas, cormos o yemas laterales, que emergen de la base de la planta principal de cultivos previos (Martínez et al. 1999; Aguas \& Martínez, 2003; Tenkouano et al. 2006; Díaz et al. 2007). Debido a esto, el material de siembra infectado ha propiciado la diseminación mundial de plagas, como el nematodo barrenador Radopholus similis (Cobb) Thorne y otros fitonematodos (Sarah et al. 1996; Gowen et al. 2005).

$R$. similis es, en la mayoría de los países donde se siembra plátano, el más abundante y constituye más del $70 \%$ de la población de nematodos en las raíces. Este fitonematodo se desarrolla y completa su ciclo de vida dentro de las raíces de las plantas afectadas (Araya, 2003) y puede producir lesiones de longitud variable ( $5 \mathrm{~cm}$ o más) en forma de estrías que, inicialmente, son de color amarillo claro, luego, rosado rojizas y, finalmente, cafés o negras. En algunos casos produce depresiones en el tejido que modifican la anatomía cilíndrica original de las raíces (Blake, 1969; Oramas \& Román, 2006). En infestaciones altas, las lesiones rodean completamente las raíces, destruyéndolas totalmente (Sarah et al. 1996; Marín et al. 1998; Gowen et al. 2005). Dicha coloración, se caracteriza por estar infestada con todos los estados de desarrollo del nematodo (Sarah et al. 1996; Marín et al. 1998; Gowen et al. 2005).

Las plantas afectadas por $R$. similis pierden anclaje por el deterioro del sistema radical, por lo cual, tienden a desraizarse o volcarse, particularmente, durante vientos y lluvias fuertes (Loos \& Loos, 1960; Sarah et al. 1996; Sarah, 2000; Araya, 2003), lo que reduce el rendimiento ( $t$ ha-1) entre 52 y 80\% (Fogain, 2000; Moens et al. 2004).

Las principales estrategias de manejo de los nematodos fitoparásitos en musáceas están determinadas por prácticas agronómicas, tales como: evitar la introducción de los nematodos en el suelo antes de establecer un cultivo, promover la sanidad y el vigor de las raíces de las plantas, reducir la oportunidad que el nematodo penetre las raíces o rizomas y utilizar material de siembra libre de fitonematodos (Loos \& Loos; 1960; Blake, 1969; Sarah, 2000; Guzmán \& Castaño, 2004; Araya, 2003; Gowen et al. 2005; Hauser, 2007).
En Colombia, los cultivos menores de 5 ha son establecidos, en su mayor parte, con cormos sin tratar; consecuentemente, estos llevan consigo problemas fitosanitarios, resultando en la contaminación de los nuevos cultivos. En algunas ocasiones, se realizan prácticas como la inmersión de los cormos en solución de aldicarb, en dosis de $10 \mathrm{~mL} \mathrm{~L}^{-1}$, en carbofuran, en dosis de $20 \mathrm{~mL} \mathrm{~L}^{-1}$, en agua caliente, a $60^{\circ} \mathrm{C}$ durante $30 \mathrm{~min}$ o en agua hirviendo a $100^{\circ} \mathrm{C}$ por $1 \mathrm{~min}$, que contribuyen a la reducción de la población de nematodos (Guzmán \& Castaño, 2004; Ramírez \& Castaño, 2009).

Debido a que uno de los métodos más sencillos y económicos para el manejo de los nematodos fitoparásitos consiste en pelar o mondar los rizomas hasta cerca de la región de mangin para remover los tejidos lesionados y erradicar los nematodos fitoparásitos, se planteó este trabajo, cuyo objetivo fue determinar el efecto de la limpieza sanitaria "pelado" o "mondado" de los cormos de plátano Dominico-Hartón, sobre el crecimiento de las plántulas y poblaciones de nematodos fitoparásitos, en condiciones de almácigo, método que hasta el momento no se ha utilizado ni recomendado en Colombia.

\section{MATERALES Y MÉTODOS}

El experimento, se realizó en la granja Montelindo, de la Universidad de Caldas, ubicada en la vereda Santágueda, municipio de Palestina, departamento de Caldas, a $5^{\circ} 05^{\prime} \mathrm{N}$ y $75^{\circ}$ $40^{\prime} \mathrm{W}$ y a 1.050 msnm. Durante el experimento, se registró una temperatura promedio anual de $23^{\circ} \mathrm{C}$ y precipitación acumulada de $1.800 \mathrm{~mm}$.

Se utilizaron cormos de plátano Dominico-Hartón, con un peso promedio de $1.357 \mathrm{~g}$. Unos, como cormos tradicionales, a los cuales, se les retiraron las raíces solamente (Figura 1A) y, otros, con limpieza sanitaria "pelado" (Figura 1B).

La limpieza sanitaria consistió en hacer cortes "pelado" con un cuchillo del tejido necrosado de los cormos afectados, principalmente, por nematodos fitoparásitos y picudos, para eliminarlos, de tal manera que al finalizar el procedimiento, se obtuvieron cormos de color crema claro. La práctica de limpieza sanitaria, en algunos cormos, se realizó máximo hasta $5 \mathrm{~cm}$ antes de la región donde se forman las raíces o región de mangin. Los cormos con daño severo que involucraba profundizar hasta la región de mangin fueron desechados. Después de realizar esta práctica, los cormos quedaron con un peso promedio de $771 \mathrm{~g}$, lo que indica que el tejido eliminado fue de aproximadamente $585 \mathrm{~g}$.

Los cormos, se sembraron en bolsas de $5 \mathrm{~kg}$ de capacidad. El llenado de las bolsas, se hizo con suelo de la misma gran- 

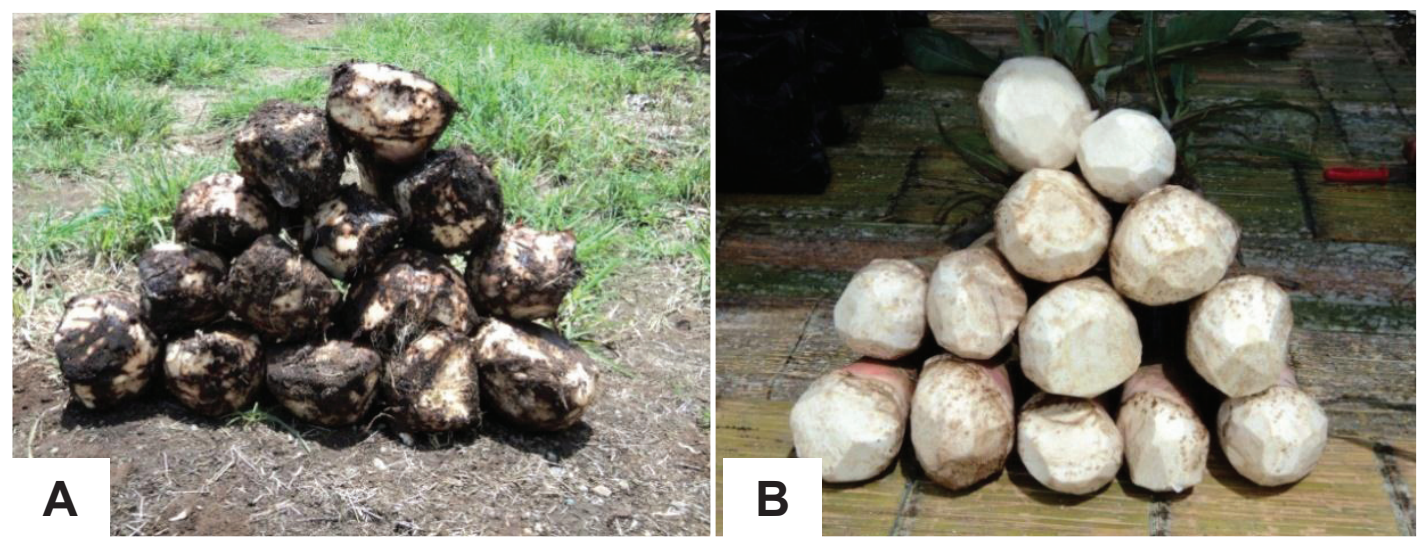

Figura 1. Material de siembra de plátano Dominico-Hartón. A. Cormo tradicional sin raíces. B. Cormos con limpieza sanitaria "pelado".

ja, originado a partir de cenizas volcánicas andesíticas de grano fino, clasificado como Tipic dystrandept con buena fertilidad natural y textura franco arenosa (Salazar \& Duque, 1994).

Para conocer el efecto de la limpieza sanitaria "pelado" de los cormos de plátano sobre el desarrollo de las plántulas y su efecto en las poblaciones de nematodos fitoparásitos, se utilizaron 120 cormos, repartidos en seis tratamientos, con 20 cormos cada uno, de la siguiente manera: los tratamientos 1 y 2, correspondieron a cormos pelados con y sin inmersión en aldicarb (Temik ${ }^{\circledR}$ 15GR, $1 \%$ durante 1 hora), respectivamente, sembrados en suelo esterilizado con dazomet (Basamid®, $50 \mathrm{~g} \mathrm{~m} 2-1$, durante 15 días); los tratamientos 3 y 4 fueron cormos tradicionales con y sin inmersión en aldicarb, respectivamente, también sembrados en suelo esterilizado con dazomet y los tratamientos 5 y 6 , correspondieron a cormos con limpieza sanitaria "pelado" con y sin inmersión en aldicarb, respectivamente, sembrados en suelo infestado naturalmente con $R$. similis.

Antes del establecimiento del experimento, se contabilizó la población inicial de nematodos fitoparásitos, tanto en el suelo esterilizado e infestado como en las raíces de las plantas de donde se obtuvieron los cormos. Para ello, se recolectaron $2 \mathrm{~kg}$ de muestra de cada uno y se trasladaron al laboratorio de Fitopatología del departamento de Producción Agropecuaria de la Universidad de Caldas, donde se realizó la extracción de los nematodos, basados en el principio de flotación en azúcar, descrito por Meredith (1973) y Araya et al. (1995).

Se obtuvieron los promedios para la variable "número de nematodos fitoparásitos', de cada género o especie. La identificación, se realizó en un microscopio de luz marca LW
Scientific Revelation III en el objetivo 40X y con las claves taxonómicas de Thorne (1961), Maggenti et al. (1987), Luc et al. (1990), Hunt (1993), Mai et al. (1996) y Siddiqi (2000).

Se utilizó un diseño experimental completamente aleatorio con 20 unidades experimentales (cormos) en cada tratamiento. A cada unidad experimental, se le aplicó riego cuando fue requerido, hasta el final del experimento, con un intervalo de 4-5 días, con el fin de mantenerla a $60 \%$ de capacidad de campo. A los 60 días después de la siembra, se realizó un muestreo destructivo de todas las plantas de cada tratamiento, para evaluar la población de fitonematodos: número de nematodos por $100 \mathrm{~g}$ de suelo y de raíces y las variables de crecimiento: porcentaje de brotación (plántulas efectivas/cormos sembrados), altura de plántulas (distancia en centímetros desde el nivel del suelo hasta la base de hoja bandera) y biomasa (materia seca de raíces de cada unidad experimental).

Para el análisis de los datos, se utilizó el programa SAS (Statistical Analysis System), con el cual, se obtuvo el análisis de varianza y las diferencias entre tratamientos, según la prueba de comparación múltiple de Tukey al $5 \%$.

\section{RESULTADOS Y DISCUSIÓN}

La población inicial de nematodos fitoparásitos resultante del análisis de suelo, antes de ser tratado con dazomet, fue de cinco individuos del género Helicotylenchus Cobb, mientras que en el suelo infestado se obtuvieron 190 individuos de $R$. similis y 246 de Helicotylenchus, en $100 \mathrm{~g}$ de suelo.

La población inicial de nematodos fitoparásitos en raíces fue de 33.390 individuos de $R$. similis y de 2.385 de Helicotylen- 
chus en $100 \mathrm{~g}$ de raíces, lo cual, evidenció la alta población de nematodos en los cormos utilizados en el experimento.

En la población de nematodos fitoparásitos, después de los tratamientos, el mejor tratamiento fue el empleo de suelo esterilizado (libre de nematodos) y cormos con limpieza sanitaria "pelado", sin presentar diferencia con el uso (tratamiento 1) o no (tratamiento 2) de aldicarb, ya que no se encontraron nematodos fitoparásitos, tanto en raíces como en suelo, después de 50 días del tratamiento, en comparación con los demás tratamientos, en los que la cantidad de nematodos fitoparásitos osciló entre 143 y 2.495 individuos en $100 \mathrm{~g}$ de raíces y entre 11 y 18 nematodos en $100 \mathrm{~g}$ de suelo (Figura 2).

En suelo esterilizado y cormos tradicionales con inmersión en aldicarb (tratamiento 3) o sin ésta (tratamiento 4), sólo se encontró $R$. similis en raíces, mientras que en suelo infestado de nematodos y cormos con limpieza sanitaria con inmersión en aldicarb (tratamiento 5) o sin ésta (tratamiento 6 ), el $79 \%$ de los fitonematodos fueron $R$. similis, el $11 \%$ Helicotylenchus y el resto Tylenchus Bastian y Aphelenchus Bastian (Figura 2).
Estos resultados coinciden con los de Loos \& Loos (1960), quienes al hacer un pelado superficial de cormos parasitados fuertemente por $R$. similis y sembrados en aserrín y suelo estériles, obtuvieron plántulas libres de nematodos fitoparásitos, después de 12 y 19 semanas del tratamiento, respectivamente.

Tenkouano et al. (2006) y Hauser (2007) afirman que los cormos de plátano y banano pelados a $5 \mathrm{~mm}$ de la capa más externa, desprovistos de raíces y tejido necrosado, están libres de nematodos fitoparásitos y picudo negro (Cosmopolites sordidus Germar). En esta investigación, el pelado se hizo hasta cerca de la región de mangin, cuya ubicación depende del tamaño del cormo, lo que demuestra que el empleo de esta práctica sanitaria evita que poblaciones de nematodos fitoparásitos sean transportadas con los cormos "semillas" infectados a áreas libres de éstos.

Cormos obtenidos de plantaciones de banano y de plátano infectadas por el nematodo Barrenador ( $R$. similis) son el principal medio de supervivencia y de dispersión de este (Loos \& Loos, 1960; Sarah et al. 1996; Araya, 2003; Coyne et al. 2003; Gowen et al. 2005). Por tal motivo, la limpieza

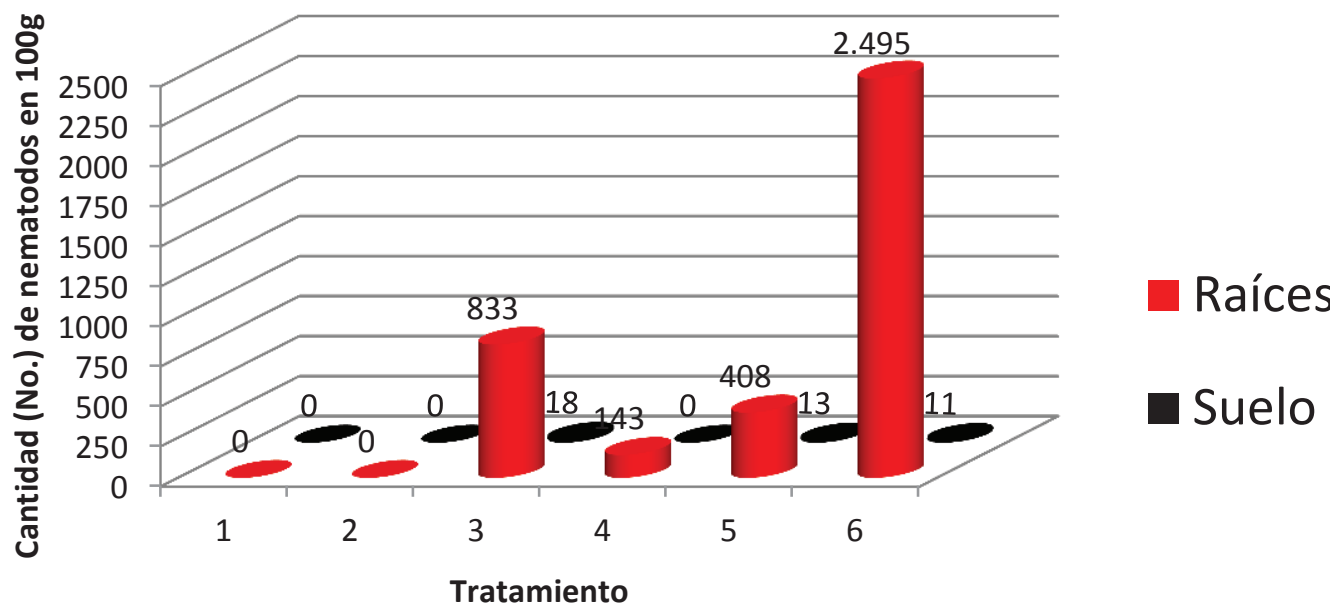

Figura 2.Cantidad de nematodos fitoparásitos en suelo y raíces después de 50 días del tratamiento. 1. Suelo esterilizado y cormos con limpieza sanitaria "pelado" con inmersión en aldicarb, 2. Suelo esterilizado y cormos con limpieza sanitaria "pelado" sin inmersión en aldicarb, 3. Cormos tradicionales con inmersión en aldicarb, 4. Cormos tradicionales sin inmersión en aldicarb, 5. Suelo infestado por nematodos fitoparásitos y cormos con limpieza sanitaria "pelado" con inmersión en aldicarb, 6. Suelo infestado por nematodos fitoparásitos y cormos con limpieza sanitaria "pelado" sin inmersión en aldicarb. 
sanitaria "pelado" de cormos de lotes infestados de nematodos fitoparásitos permite obtener plántulas libres de estos (Fogain, 2000; Tenkouano et al. 2006; Hauser, 2007), como queda confirmado en esta investigación.

El sistema radical de las plántulas con limpieza sanitaria "pelado" o "mondado" (tratamientos 1 y 2) fue completamente blanco, indicando raíces sanas y libres del nematodo Barrenador ( $R$. similis), como se indicó anteriormente. Así mismo, el sistema radical de las plántulas obtenidas de cormos tradicionales y sembrados en suelo estéril (tratamientos 3 y 4), presentó algunas raíces con necrosis y las plántulas provenientes de cormos con limpieza sanitaria y sembrados en suelo infestado de nematodos fitoparásitos (tratamientos 5 y 6), mostraron raíces necróticas y de coloración violeta. De estas raíces, se extrajo el nematodo espiral Helicotylenchus spp., el cual, ha sido asociado a dicha sintomatología, por Gowen et al. (2005), quienes afirman que $H$. multicinctus (Cobb) Golden produce lesiones color marrón rojizo a negro en banano y plátano.

Según Díaz et al. (2007), el cormo de las musáceas funciona, inicialmente, como reserva temporal de nutrimentos para la nueva planta, que emergerá e iniciará su desarrollo, dependiendo de dichas reservas, mientras ocurre la emisión de raíces suficientes, que le permita absorber agua y nutrimentos del suelo, requeridos para su desarrollo. Al eliminar el tejido dañado de los cormos, también se elimina tejido sano, que sirve de fuente de nutrimentos, lo que reduce viabilidad y vigor de las plántulas que emergen, con alta probabilidad de morir antes de desarrollar un sistema radicular apropiado. Estas afirmaciones no coinciden con los resultados de esta investigación, debido a que los cormos con limpieza sanitaria "pelado", presentaron una excelente brotación y, además, las plántulas tuvieron un apropiado desarrollo del sistema radical y aéreo (Figura 3). Debido a que los cormos estaban libres de nematodos, aunque la brotación fue muy buena, ésta ocurrió ocho días después de la brotación de los cormos tradicionales.

Cuando el cormo está afectado por $R$. similis, la remoción de tejidos contaminados, mediante limpieza sanitaria "pelado", es una práctica sencilla, eficaz y de fácil adopción por los agricultores para el manejo de este nematodo (Loos \& Loos; 1960; Blake, 1969; Sarah, 2000; Gowen et al. 2005; Hauser, 2007).

Los resultados anteriores demuestran que la limpieza sanitaria "pelado" de los cormos permite obtener plántulas libres de nematodos fitoparásitos, como el nematodo Barrenador (R. similis), lo cual, favorece el crecimiento y el desarrollo de las plántulas e incrementa la producción.

En relación con las variables de crecimiento, se obtuvo una brotación alta de los cormos en todos los tratamientos, que osciló entre 90 y 100\%. Esta última, se obtuvo utilizando
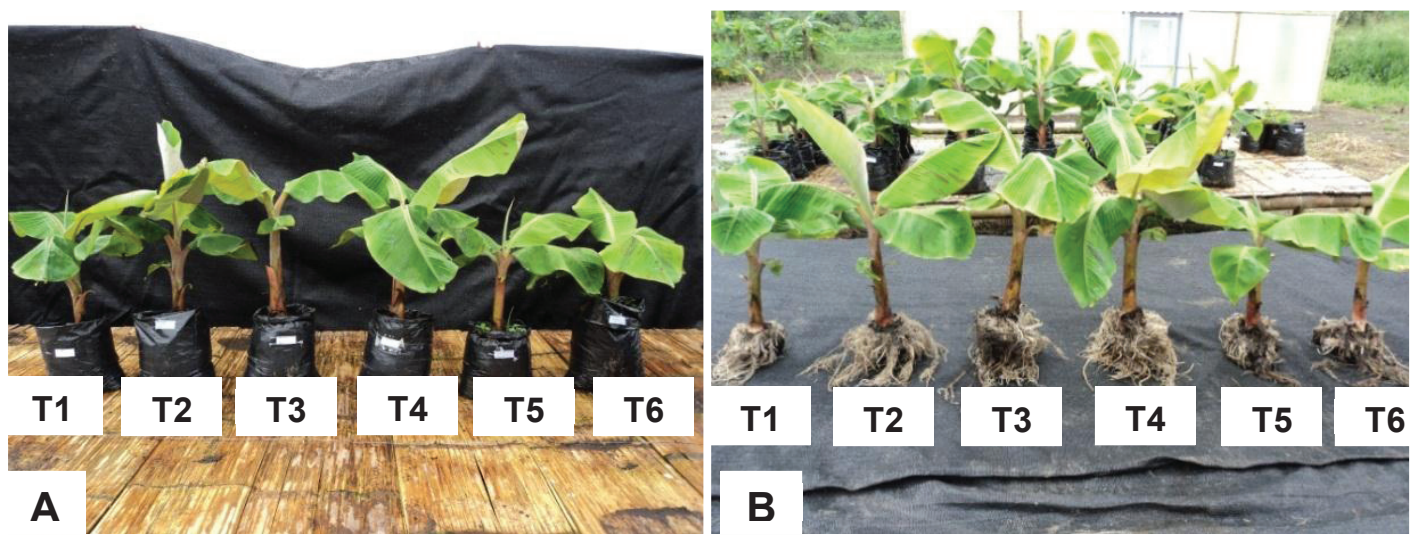

Figura 3. Plántulas de plátano Dominico-Hartón (Musa AAB) en bolsas de almácigo (A) y con raíces desnudas (B), después de 50 días del tratamiento. 1. Suelo esterilizado y cormos con limpieza sanitaria "pelado" con inmersión en aldicarb, 2. Suelo esterilizado y cormos con limpieza sanitaria "pelado" sin inmersión en aldicarb, 3) Cormos tradicionales con inmersión en aldicarb, 4. Cormos tradicionales sin inmersión en aldicarb, 5. Suelo infestado por nematodos fitoparásitos y cormos con limpieza sanitaria "pelado" con inmersión en aldicarb, 6. Suelo infestado por nematodos fitoparásitos y cormos con limpieza sanitaria "pelado" sin inmersión en aldicarb. 
suelo tratado con el fumigante dazomet más cormos con limpieza sanitaria "pelado" y cormos convencionales, ambos tratados con el nematicida aldicarb.

Referente a la materia seca de raíces, no se encontraron diferencias estadísticas significativas entre el sistema de raíces de las plántulas provenientes de cormos con limpieza sanitaria "pelado" y los cormos tradicionales (Figura 4). Es de resaltar los tratamientos 5 y 6 (suelo infestado por nematodos fitoparásitos y cormos con limpieza sanitaria "pelado" con y sin inmersión en aldicarb, respectivamente), donde la materia seca presentó los valores más bajos (Figura 4), atribuidos a la presencia de $R$. similis y Helicotylenchus spp., que afectaron el crecimiento del sistema radical de las plántulas.

En cuanto a la materia seca de la parte aérea de las plántulas, los tratamientos 2, 3 y 4 con suelo esterilizado y cormos con limpieza sanitaria "pelado" sin inmersión en nematicida y los tratamientos en suelo esterilizado y cormos tradicio- nales con y sin inmersión en nematicida, respectivamente, fueron los que presentaron el mayor contenido de materia seca, con un peso promedio de $13,86 \mathrm{~g}$, sin presentar diferencias estadísticas significativas entre ellos, pero sí con los demás tratamientos. (Figura 4). Esto se explica, porque los cormos tradicionales no presentan daño mecánico por acción del pelado y, para el caso del cormo pelado, el efecto de la sanidad también contribuye al buen desarrollo y no afecta el contenido de materia seca. Aunque el tratamiento 1 (suelo esterilizado y cormos con limpieza sanitaria "pelado" con inmersión en aldicarb) presentó diferencias estadísticas con los tratamientos que obtuvieron el mayor peso en materia seca de la parte aérea, el peso promedio de materia seca fue de $12 \mathrm{~g}$, similar al promedio de los mejores tratamientos arriba mencionados.

Tal como se obtuvo en los resultados de materia seca de raíces, los tratamientos 5 y 6 presentaron el menor contenido de materia seca aérea, con un peso promedio de 7,89g (Fi-

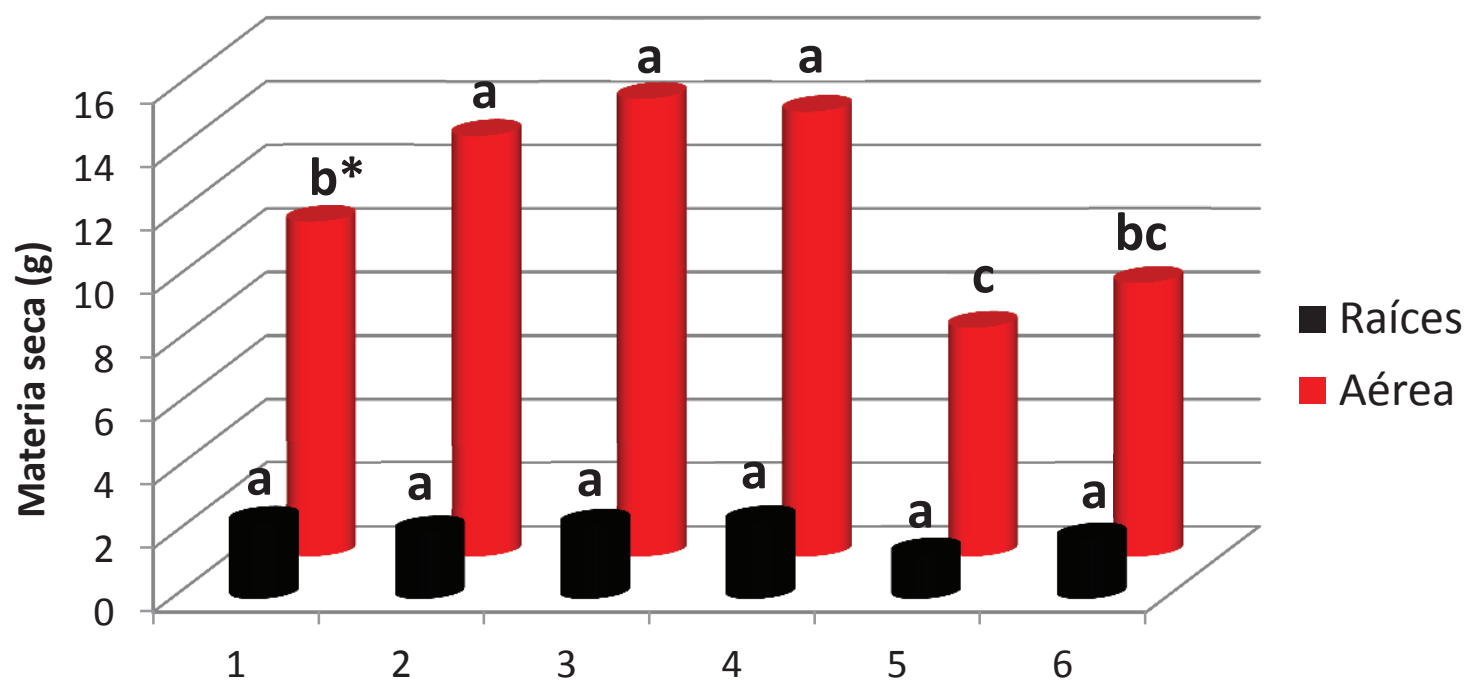

Tratamiento

Figura 4. Materia seca de raíces y parte aérea de plántulas de plátano Dominico-Hartón, después de 50 días del tratamiento. 1. Suelo esterilizado y cormos con limpieza sanitaria "pelado" con inmersión en aldicarb, 2. Suelo esterilizado y cormos con limpieza sanitaria "pelado" sin inmersión en aldicarb, 3. Cormos tradicionales con inmersión en aldicarb, 4. Cormos tradicionales sin inmersión en aldicarb, 5. Suelo infestado por nematodos fitoparásitos y cormos con limpieza sanitaria "pelado" con inmersión en aldicarb, 6. Suelo infestado por nematodos fitoparásitos y cormos con limpieza sanitaria "pelado" sin inmersión en aldicarb.

*Las columnas seguidas por la misma letra no se diferencian significativamente, de acuerdo a la prueba de comparación múltiple de Tukey al $5 \%$. 
gura 4), atribuido a la presencia de $R$. similis y Helicotylenchus spp., que afectaron el crecimiento y desarrollo aéreo de las plántulas (Figura 3).

Moens et al. (2003) encontraron una reducción lineal en el peso de raíces de Musa AAA cv. Grande Naine cuando $R$. similis fue inoculado. Como consecuencia, el peso de las raíces disminuyó en 3,9g (16\%) por cada inoculación sucesiva de 1.000 nematodos. Sarah et al. (1993) hallaron que después de ocho semanas de exponer plántulas de plátano Valery a una población de 300 individuos de $R$. similis, el peso de las raíces se disminuyó entre 19 y $80 \%$. Así mismo, Hahn et al. (1996), empleando la misma cantidad de individuos de este nematodo y en la misma variedad, encontraron, después de doce semanas, una reducción en el peso de las raíces superior al $30 \%$.

Respecto a la altura de las plántulas, se hallaron resultados similares a los obtenidos con la materia seca aérea, en donde el uso de suelo esterilizado y cormos con limpieza sani- taria "pelado" sin inmersión en nematicida (tratamiento 2) y cormos tradicionales con y sin inmersión en nematicida (tratamientos 3 y 4) fueron los que presentaron la mayor altura con un promedio de $24,84 \mathrm{~cm}$, sin presentar diferencias estadísticas significativas entre ellos (Figura 5).

Por el contrario, las plántulas que presentaron menor altura fueron aquellas provenientes del empleo de suelo infestado por nematodos fitoparásitos y cormos con limpieza sanitaria "pelado" con y sin inmersión en aldicarb, cuya altura promedio fue de $16,41 \mathrm{~cm}$, es decir, $8 \mathrm{~cm}$ menos que en los tratamientos con suelo libre de nematodos fitoparásitos.

Como resultado del ataque de los nematodos fitoparásitos, principalmente $R$. similis, Fogain (2000), en Njombe (Camerún), encontró que las plantas parasitadas por este nematodo tuvieron una altura menor de 21 y $18 \mathrm{~cm}$, en comparación con las plantas tratadas con nematicidas en el primero y segundo ciclo productivo, respectivamente.

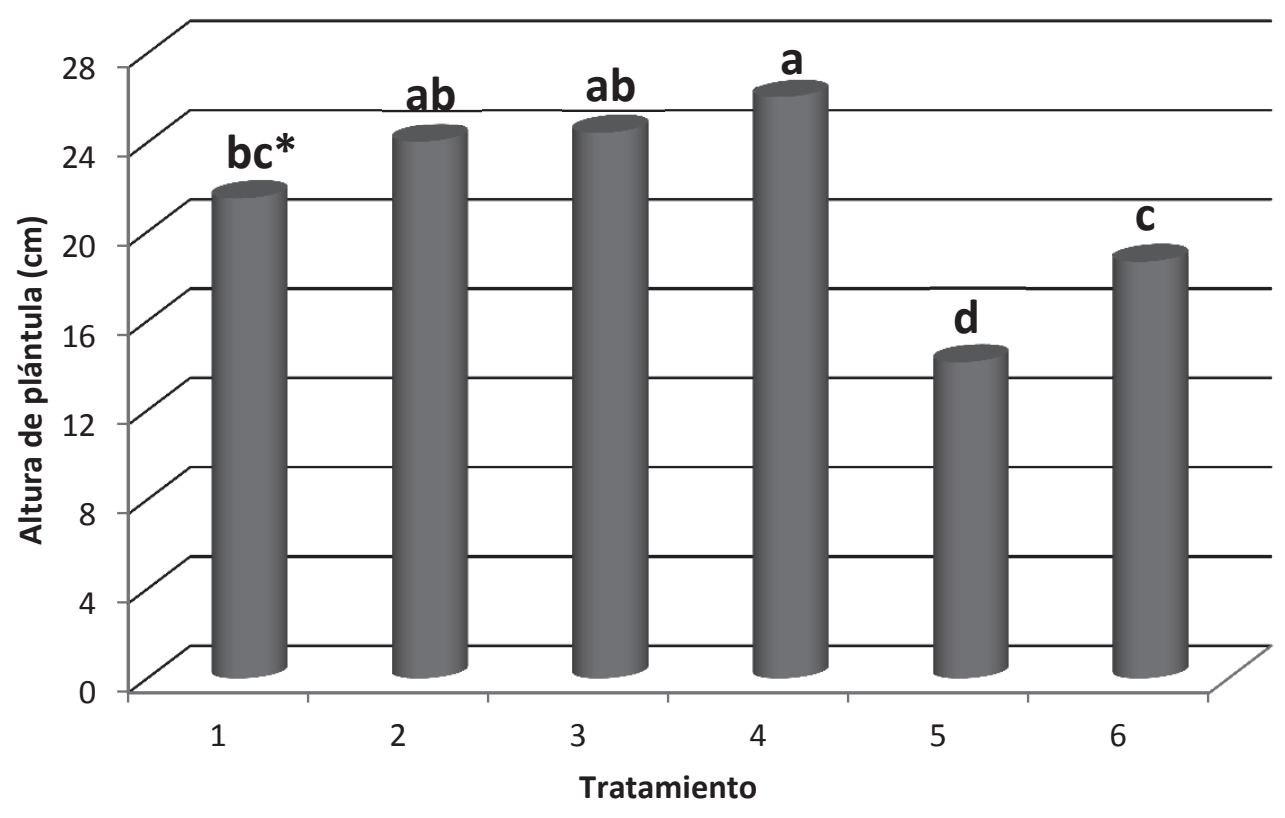

Figura 5. Altura $(\mathrm{cm})$ de plántulas de plátano Dominico-Hartón (Musa AAB) después de 50 días del tratamiento. 1. Suelo esterilizado y cormos con limpieza sanitaria "pelado" con inmersión en aldicarb, 2. Suelo esterilizado y cormos con limpieza sanitaria "pelado" sin inmersión en aldicarb, 3. Cormos tradicionales con inmersión en aldicarb, 4. Cormos tradicionales sin inmersión en aldicarb, 5. Suelo infestado por nematodos fitoparásitos y cormos con limpieza sanitaria "pelado" con inmersión en aldicarb, 6. Suelo infestado por nematodos fitoparásitos y cormos con limpieza sanitaria "pelado" sin inmersión en aldicarb.

*Las columnas seguidas por la misma letra no se diferencian significativamente, de acuerdo a la prueba de comparación múltiple de Tukey al $5 \%$. 
La limpieza sanitaria, pelado o mondado de cormos, permitió obtener plántulas de plátano Dominico-Hartón libres de nematodos fitoparásitos, como el nematodo Barrenador $(R$. similis), al igual que un mayor contenido de materia seca, lo cual, permite llevar al campo material de propagación sano e incrementar la producción del cultivo.

En Colombia, la práctica de limpieza sanitaria de cormos puede ser fácilmente adoptada por los cultivadores de plátano y de banano, debido a que es sencilla, económica y no contamina el medio ambiente; por lo tanto, se recomienda incluirla en el manejo integrado de plagas, como nematodos fitoparásitos y picudos.

Agradecimientos. Este estudio fue financiado por la Vicerrectoría de Investigaciones y Postgrados de la Universidad de Caldas. Conflicto de intereses: Los autores declaramos que no existe ningún conflicto de intereses que ponga en riesgo la validez de los resultados presentados.

\section{BIBLIOGRAFÍA}

1. AGUAS, A.A.; MARTíNEZ, M. 2003. Técnicas rápidas para la multiplicación de semillas de plátano. Boletines Divulgativos Ecorregión Caribe (COL), (69), 7p.

2. ARAYA, M.; CENTENO, M.; CARRILLO, W. 1995. Densidad poblacional y frecuencia de los nematodos parásitos de banano (Musa AAA) en nueve cantones de Costa Rica. CORBANA (Costa Rica). 20(43):6-11.

3. ARAYA, M. 2003. Situación actual del manejo de nematodos en banano (Musa AAA) y plátano (Musa $A A B)$ en el trópico americano. En: Rivas G.; Rosales, F. eds. Manejo Convencional y alternativo de la Sigatoka Negra, Nematodos y Otras Plagas Asociadas al Cultivo de Musáceas en los Trópicos. INIBAP, Francia.p.79-102.

4. BLAKE, C.D. 1969. Nematode parasites of banana and their control. In: Peachy, J.E. (Ed). Nematodes of Tropical Crops. Technical Communication No. 40.St Albans, UK, Commonwealth Bureau of Helminthology. p.109-132.

5. COYNE, D.; KAJUMBA, C.; KAGODA, F. 2003. Nematode management at the International Institute of Tropical Agriculture. In: Blomme, G.; Gold, C.; Karamura, E. (eds.), Farmer-Participatory Testing of Banana Integrated Pest Management Options for Sustainable Banana Production in Eastern Africa, Proc. Workshop on Farmer-participatory testing of IPM op- tions for sustainable banana production in Eastern Africa, held in Seeta, Uganda, 8-9 December 2003, INIBAP, Montpellier, France. p.141-148.

6. DÍAZ, M.J.; RIVERA, C.M.; DURÁN, L. 2007. Como proteger de las plagas del suelo los cormos-semilla de plátano y banano. 1a ed. La Lima, Cortés, Honduras: FHIA, Departamento de Protección Vegetal. 18 p.

7. FOGAIN, R. 2000. Effect of Radopholus similis on plant growth and yield of plantain (Musa AAB). Nematology. 2:129-133.

8. GOWEN, S.; QUÉNÉHERVÉ, P.; FOGAIN, R. 2005. Nematodes parasites of bananas and plantains. Chapter 16: En: Luc, M.; Sikora, J.; Bridge, J. (eds). Plant Parasitic Nematodes in Subtropical and Tropical Agriculture. p.611-643.

9. GUZMÁN, O.; CASTAÑO, J. 2004. Reconocimiento de nematodos fitopatógenos en Dominico Hartón (Musa AAB Simmonds) África, FHIA 20 y FHIA 21 en la granja Montelindo, municipio de palestina (Caldas), Colombia. Rev. Acad. Col. Cienc. Ex., Fís. Nat. 38(107):295-301.

10. HAHN, M.L.; SARAH, J.L.; BOISSEAU, M.; VINES, N.J.; WRIGHT, D.J.; BURROWS, P.R. 1996. Reproductive fitness and pathogenicity of selected Radopholus similis populations on two banana cultivars. Plant Path. 45:223-231.

11. HAUSER, S. 2007. Plantain (Musa spp. AAB) bunch yield and root health response to combinations of physical, thermal and chemical sucker sanitation measures. Afr. Plant Prot. 13:1-15.

12. HUNT, D.J. 1993. Aphelenchida, Longidoridae and Trichodoridae: Their Systematics and Bionomics. CABI Bookshop. 368p.

13. LOOS, C.A.; LOOS, S. 1960. Preparing nematode-free banana "seed". Phytopath. 50:383-386.

14. LUC, M.; SIKORA, R.; BRIDGE, J. 2005. Plant Parasitic nematodes in subtropical and tropical agriculture. $2^{\text {nd }}$ Edition. 871p.

15. LUC, M.; HUNT, D.; MACHON, J. 1990. Morphology, Anatomy an dBiology of plant parasitic nematodes a Synopsis. En: Luc, M.; Sikora, R.; Bridge, J. (eds). Plant Parasitic Nematodes in Tropical Agriculture. CAB International. London. p.1-44. 
16. MAGGENTI, A.; LUC, M.; RASKI, D.; FORTUNER, R.; GERAERT, E. 1987. A Reappraisal of Tylenchina (Nemata). 2. Classification of the suborder Tylenchina (Nemata: Diplogasteria). Revue Nématol. 10(2):135-142.

17. MAI, W.; MULLIN, P.; LYON. H.; LOEFFLER, K. 1996. Plant parasitic nematodes. A pictorical key to genera. Fifth edition. Comstock Publ. Assoc. A Division of Cornell University Press. 277p.

18. MARÍN, D.; SUTTON, T.B.; BARKER, K.R. 1998. Dissemination of bananas in Latin America and the Caribbean and its relationship to the occurrence of Radopholus similis. Plant Dis. 82(9):964-974.

19. MARTÍNEZ, G.; MANZANILLA, E.; PARGAS, R. 1999. Modelo de propagación y producción simultanea en musáceas. FONAIAP Divulga (VEN.). 64:2-6.

20. MEREDITH, J. 1973. Algunos métodos de campo y laboratorio para trabajar con nematodos. Maracaibo (Venezuela). 44p.

21. MOENS, T.; ARAYA, M.; SWENNEN, R.; DE WAELE, D.; SANDOVAL, J. 2003. Growing medium, inoculum density, exposure time and pot volume: factors affecting the resistance screening for Radopholus similis in banana (Musa spp.). Nematropica 33(1):926.

22. MOENS, T.; ARAYA, M.; SWENNEN, R.; DE WAELE, D. 2004. Enhanced biodegradation of nematicides after repetitive applications and its effect on root and yield parameters in commercial banana plantations. BioFertilSoils 39:407-414.

23. ORAMAS, D.; ROMÁN, J. 2006. Histopatología de los nematodos Radopholus similis, Pratylenchus coffeae, Rotylenchulus reniformis y Meloidogyne incognita en plátano (Musa acumulata X M. balbisiana, AAB). J. Agric. Univ. P.R. 90(1-2):83-97.
24. RAMÍREZ, C.; CASTAÑO, J. 2009. Efecto del tratamiento químico de la semilla de plátano con diferentes productos y tiempos de exposición sobre la población de nematodos fitoparásitos. Fitotecnia. 144:2.

25. SALAZAR, H.; DUQUE, S. 1994. Manejo cultural y químico de la pudrición acuosa del pseudotallo (Erwinia chrysantemi pv. paradisiaca) en Musa sp. Fitopat. Col. 18(1):20-35.

26. SARAH, J.L. 2000. Burrowing nematode. In: Jones, D.R. (ed.) Diseases of banana, Abacá and Enset. CABI Publishing. Wallingford, UK. p.295-303.

27. SARAH, J.L.; PINOCHET, J.; STANTON, J. 1996. El nematodo barrenador del banano Radopholus similis Cobb. Plagas de Musa - INIBAP, Francia.Hoja Divulgativa No. 1. 2p.

28. SARAH, J.L.; SABATINI, C.; BOISSEAU, M. 1993. Differences in pathogenicity to banana (Musa sp. cv. Poyo) among isolates of Radopholus similis from different production areas of the world. Nematropica. 23:7379.

29. SIDDIQI, M.R. 2000. Tylenchida: Parasites of plants and insects, 2nd Edition. CABI Bookshop. 834p.

30. TENKOUANO, A.; HAUSER, S.; COYNE, D.; COULIBALE, O. 2006. Clean planting materials and management practices for sustained production of banana and plantain in Africa. Chron. Hort. 46:1418.

31. THORNE, G. 1961. Principles of nematology. New York, McGraw Hill, Book Company. 553p.

Recibido: Octubre 10 de 2011

Aceptado: Abril 19 de 2012 